EDITORIAL

\title{
Innovative approaches in humanitarian operations
}

\author{
Walter J. Gutjahr ${ }^{1} \cdot$ Nilay Noyan ${ }^{2} \cdot$ Nico Vandaele $^{3} \cdot$ Luk N. Van Wassenhove $^{4}$
}

Received: 11 July 2020 / Accepted: 13 July 2020 / Published online: 27 July 2020

(c) Springer-Verlag GmbH Germany, part of Springer Nature 2020

Natural or man-made disasters, humanitarian crises, epidemics and other emergency situations confront governmental institutions and humanitarian organizations with enormous challenges. Fast decisions under uncertainty have to be made, often under scarce resources, while well-being and lives of humans are at stake. The current Covid-19 pandemic makes it clear to everybody in the world that the "normal" functioning of our societies can always be threatened by disruptive events. Forceful and intelligent joint efforts are required to avert the most serious consequences of disasters of different types.

Humanitarian operations can be supported by traditional techniques of engineering, information technology and logistics management, but these approaches frequently turn out to be insufficient in disaster environments. The field of Operations Research can contribute to overcome some of the limitations of current humanitarian aid.

The present Special Issue contains eight articles that investigate the application of novel approaches, be it on the side of modeling, computational solution procedures, technologies or analytics, to the area of disaster management and humanitarian operations.

Briskorn et al. (2020) study a multi-period problem of scheduling the debris removal tasks for blocked roads and planning the relief distribution operations in case of a disaster while taking into consideration the strict deadlines for fulfilling

Walter J. Gutjahr

walter.gutjahr@univie.ac.at

Nilay Noyan

nnoyan@sabanciuniv.edu

Nico Vandaele

nico.vandaele@kuleuven.be

Luk N. Van Wassenhove

luk.van-wassenhove@insead.edu

1 University of Vienna, Oskar-Morgenstern-Platz 1, 1090 Vienna, Austria

2 Sabanci University, Istanbul, Turkey

3 KU Leuven, Leuven, Belgium

4 INSEAD Fontainebleau, Fontainebleau, France 
demand at each location. In particular, the problem simultaneously determines the blocked edges to clear and the delivery amount from each supply node to each demand node in each period, while minimizing the duration of road clearance. The proposed model represents each blocked road by an integer number of blocked edges in a network, where clearing one blocked edge is assumed to take one period. Accordingly, the objective is to find a minimum length sequence of blocked edges to clear so that all demand can be satisfied before their corresponding deadlines. The authors provide two equivalent statements of the problem of interest, which differ in their graph representations, along with some computational complexity results. Focusing on the variant with a smaller graph representation, referred to as the reduced problem, the authors develop a mixed-integer programming (MIP) formulation and an exact branch-and-bound solution algorithm. The numerical results demonstrate the superior performance of the branchand-bound algorithm compared to directly solving the MIP formulation and provide insights about the key factors, including the density of graph, which have a significant impact on the performance of the algorithm. One future research area is the additional consideration of multiple work teams for road clearance and cooperation between them.

Ekici and Ozener (2020) address the problem of last mile delivery in the aftermath of an event requiring humanitarian assistance. In particular, the authors focus on the routing operations for the relief supplies that are consumed regularly, and accordingly, they aim to find a plan with multiple deliveries to the demand locations-also called dispensing sites. The delivery network consists of a capacitated distribution center, which receives daily shipments of a single commodity and from which a number of homogeneous capacitated vehicles are dispatched every day, and multiple capacitated dispensing sites with constant consumption rates of the commodity. The problem of interest is a variant of the well-known inventory routing problem, which mainly determines the clusters of dispensing sites served by each vehicle on each day, daily delivery routes and volumes with an equity-based objective of maximizing the minimum slack-defined as the minimum inventory level divided by the consumption rate-over all dispensing sites. The proposed mathematical programming-based framework considers the interdependence between the decisions on routing, clustering, and resources allocation to make these decisions in an integrated manner. A sequential heuristic solution approach is proposed, where the delivery amounts are estimated first, followed by the clustering of sites and routing of vehicles, with an improvement phase at the end. The authors implement two variants (the capacitated concentrator location heuristic and the integrated clustering heuristic) of the proposed solution framework. The first variant makes the clustering and routing decisions separately, while the second variant follows an integrated approach considering the interaction between clustering and delivery scheduling decisions. The computational study demonstrates the effectiveness of the proposed solution framework by benchmarking against an upper bounding scheme on randomly generated instances. This study does not address the uncertainty inherent in the network. Acknowledging this issue, the authors point out the incorporation of uncertainties in the transportation infrastructure and demand/consumption rates as an interesting future direction. 
Huertas et al. (2020) deal with a problem in emergency evacuation. Their approach adopts an optimization model for finding optimal evacuation dynamics in an area with a complex topology. A static network is expanded over time such that a dynamic (time-space) network is produced. Bi-directional flow rates and capacities are estimated; in particular, these quantities also respect the steepness of arcs, e.g., of stairways. A linear programming model determines the numbers of people flowing through each dynamic arc during the evacuation. The use of linear programming duality allows the identification of congestion hot spots. When conducting an evacuation exercise, planners face the problem that the distribution of evacuees over the evacuation area in the case of a real emergency is not known. Thus, for the mock drill, a certain number of suitable scenarios have to be created. The presented approach proposes to do this by a combination of simulation with the above-mentioned optimization model. The authors implemented their modeling framework and furnished it with a GIS-based, rich visualization function showing the evacuation dynamics. Two case studies involving the campus of the Universidad de los Andes in Bogota conclude the paper, one providing a comparison against an evacuation drill, the other one modeling an evacuation scenario derived from a real-life event in 2016 where the center of Bogota was threatened by the smoke of a nearby wildfire.

Khara et al. (2020) address a problem frequently occurring during the onset of "forecasted disasters" as hurricanes: when such a disaster is approaching, people tend to panic-buy and to hoard essential commodities, which can entail serious shortages. For adequate decision making, it is important that shortages of this kind can be predicted some time in advance. The authors focus on gasoline shortages in the course of the landfall of a hurricane and propose a novel methodology where social media posts (tweets) are used to forecast, from day to day, the number of gasoline stations out of gas in each given city. The number of tweets related to gasoline shortages is viewed as a variable that can itself be predicted from basic data. For applying the methodology, the authors developed a tweet classifier based on a support vector machine. The Poisson regression method and time series analysis by ARIMA were combined in a hybrid loss function model to achieve more accurate predictions. Khare et al. assessed their approach by an application to data on the Hurricane Irma (Florida, 2017), which included the analysis of more than one million of tweets. The article illustrates the benefits that disaster management can expect from data analytics, but the authors also go beyond the purely statistical context by discussing how their approach can be applied in an optimization framework.

Naqvi et al. (2020) focus on droughts caused by climate change, a major source of humanitarian crises. They deal with risk assessment of events where by crop failures in food-producing regions, cascading effects are triggered. Three methodological approaches are combined: copula, multi-layer networks and agent-based models. Copulas model nonlinear co-dependencies of risks. Multi-layer networks show how regions interact on different "layers", e.g., a production layer (trade) and a population layer (migration). Agent-based models, finally, allow it to represent behavioral rules governing socioeconomic interactions. The authors introduce a new risk measure, "Vulnerability Rank", which can be used, among others, to identify highly vulnerable locations that are in special need of humanitarian aid, or to study the effects of possible prevention or mitigation policies by simulation. The authors conduct a 
case study with data from India. They mention that their approach is not limited to droughts, but can also be applied to energy issues or to the spread of diseases.

Shiri et al. (2020) study a routing problem for search-and-rescue (SAR) operations involving multiple heterogeneous teams after a disaster. The decisions regarding the dispatching of the SAR teams must be made quickly under incomplete information about the key uncertain input parameters such as the conditions of the roads. In the proposed setting, the information about the number of victims at a critical node and the time necessary to rescue all the victims is revealed when a team reaches the critical node, while a blocked edge is identified when a team traverses that road segment. Accordingly, the authors develop two types of deterministic online strategies to determine the decisions of interest under the incomplete information, which is assumed to be revealed incrementally as the actions of the SAR teams are executed according to the determined online strategy. In particular, the manuscript presents the MIPs for two variants of the underlying offline problem (without any unknown parameters). The two problem variants of interest differ in the objective function: the "makespan minimization variant" focuses on the completion time of the SAR operations, while the "total weighted latency minimization" variant focuses on the average waiting time of the victims. The authors propose two heuristic solution approaches to determine an online strategy; one relies on solving an MIP formulation of the offline problem, while the other one is based on greedy choices. The numerical results illustrate the computational effectiveness of the greedy heuristic strategies on randomly generated problem instances. The authors also provide bounds on the solution quality of online problems versus the offline variant, namely lower bounds on the competitive ratio of online deterministic strategies. From a theoretical point of view, investigating the tightness of the proposed lower bounds and deriving the competitive ratio of the proposed strategies are among future endeavors.

Wankmüller et al. (2020) try to improve the emergency response system for sudden cardiac arrests (SCAs) in mountainous regions by means of defibrillator drones. A large percentage of lethal touristic events in the European Alps is caused by SCAs. In many cases, death could be prevented by a reduction in the response time required to transport an automated external defibrillator (AED) to the scene, which is currently done by a helicopter within comparable long response times. The authors suggest to use drones, located at a set of base stations, for the transportation of AEDs. They formulate an integer linear program of the type of an uncapacitated facility location problem to optimize the positions of the base stations. Two currently available types of drones with different speeds and technical properties are considered, and respective flight times between base stations and potential emergency locations are computed. In a case study for the Val Venosta region in South Tyrol, Italy, it is shown that the solutions of the facility location problem achieve dramatic reductions of the response times, compared to the standard system working with helicopters.

Warnier et al. (2020), finally, address the problem that in the aftermath of a disaster, pre-disaster road maps do not adequately represent the transportation network anymore, which makes techniques for a quick update urgently required. Even after the disaster, the network may still be rapidly changing, and information on its status 
is subject to high uncertainty. On the other hand, geographic situational awareness is a critical success factor for disaster response. The authors propose an information system that not only quickly evaluates the quality of road segments, but also analyzes the reachability of communities by techniques from network analysis. In particular, centrality measures as node-betweenness and edge-betweenness are used, and the most critical nodes and edges (with highest betweenness centrality) are visualized. The information system is able to generate an initial map of the affected area within a few hours after the disaster. A case study referring to the 2018 earthquake in Papua New Guinea illustrates the approach. The output of the system can feed data into diverse operations research models studied in the humanitarian logistics literature, e.g., facility location, debris removal, last mile distribution or vehicle routing, and contributes in this way essentially to an implementation of OR techniques in decision support systems for disaster relief.

\section{References}

Briskorn D, Kimms A, Olschok D (2020) Simultaneous planning for disaster road clearance and distribution of relief goods. OR Spectrum

Ekici A, Ozener OO (2020) Inventory routing for the last mile delivery of humanitarian relief supplies. OR Spectrum

Huertas JA, Duque D, Segura-Duran E, Akhavan-Tabatabaei R, Medaglia AL (2020) Evacuation dynamics: a modeling and visualization framework. OR Spectrum

Khara A, He Q, Batta R (2020) Predicting gasoline shortage during disasters using social media. OR Spectrum

Naqvi A, Gaupp F, Hochrainer-Stigler S (2020) The risk and consequences of multiple breadbasket failures. OR Spectrum

Shiri D, Akbari V, Salman FS (2020) Online optimization of search and rescue logistics with multiple teams. OR Spectrum

Wankmüller C, Truden C, Korzen C, Hungerländer P, Kolesnik E, Reiner G (2020) Base station allocation of defibrillator drones in mountainous regions. OR Spectrum

Warnier M, Alkema V, Comes T, van de Walle B (2020) Humanitarian access, interrupted: dynamic near real-time network analytics and mapping for reaching communities in disaster-affected countries. OR Spectrum

Publisher's Note Springer Nature remains neutral with regard to jurisdictional claims in published maps and institutional affiliations. 